CORRECTION

\title{
Lyme borreliosis
}

Allen C. Steere, Franc Strle, Gary P. Wormser, Linden T. Hu, John A. Branda, Joppe W. R. Hovius, Xin Li and Paul S. Mead

Nature Reviews Disease Primers 2, 16090 (2016)

In the version of the article originally published, it was incorrectly stated that Figure $3 \mathrm{~b}$ is a 3D model generated by cryo-electron tomography. This has now been corrected. 